\title{
Group Acceptance Sampling Plans for Resubmitted Lots UnderType II Generalized Half Logistic Distribution
}

\author{
G. S. Rao ${ }^{1 *}$, S. Jilani ${ }^{2}$, A. V. Rao $^{2}$ \\ ${ }^{1}$ Department of Statistics, The University of Dodoma, Dodoma, PO. Box: 259, Tanzania \\ ${ }^{2}$ Department of Statistics, Acharya Nagarjuna University, A. P., India
}

Received 7 December 2019, accepted in final revised form 2 February 2020

\begin{abstract}
In this research article, we build-up a group acceptance sampling plan (GASP) for a resubmitting lot when the lifetime of a product follow the Type II Generalized half-logistic distribution (GHLD). The design parameters of the group acceptance sampling plan for the resubmitted are determined by fixing the experiment termination time and the numbers of testers at the stated producer's and consumer's risks both satisfy at the same time. We contrast the proposed GASP with the ordinary group sampling plan and the results are demonstrated with a live data set.
\end{abstract}

Keywords: Producer's risk; Consumer's risk; Resubmitted lot; Acceptance sampling plan; Type II generalized half-logistic distribution.

(C) 2020 JSR Publications. ISSN: 2070-0237 (Print); 2070-0245 (Online). All rights reserved. doi: http://dx.doi.org/10.3329/jsr.v12i3.44406

J. Sci. Res. 12 (3), 311-325 (2020)

\section{Introduction}

In statistical quality control, the acceptance sampling plans are playing an important role in making verdict whether the lot under inspection is accepted or rejected. During the Second World War, acceptance sampling plans were used frequently, for instance MILSTD-105, developed by Dodge and some others, used as quality of standard. The most commonly used acceptance sampling is the single attribute acceptance sampling among all the acceptance sampling methods because it is easy to implement in practice. In single attribute acceptance sampling, the inference about acceptance or rejection of a submitted lot is based on the truncated life test. One must follow the truncated or destructive life test to examine the lifetime of a product. Considerably long time of experiment is required to observe the complete or absolute lifetime of a high reliability product. The life test which completes the test within a pre-specified schedule is known as truncated life test. Under a truncated life test, single acceptance sampling plans have been explored few decades in the past [1-12].

\footnotetext{
*Corresponding author: gaddesrao@gmail.com
} 
In a life test to save time and cost, it is very often, we put a number of items in a group, usually known as a tester and the number of items in each tester is called the group size. The acceptance sampling plan, which is based on group life test, is known as the group acceptance sampling plan (GASP) and is implemented under a truncated life test also. Since the inception of the concept of group into acceptance sampling plan [13], GASPs based on truncated life tests have been studied by several authors for different lifetime distributions; and developed variable sampling plans for the Weibull distribution for sudden death testing. For instance, Aslam proposed the GASP under the time truncated experiment assuming the product lifetimes follows the Weibull distribution [14]. The author also developed the GASPs, using single point on operating characteristics (OC) curve, for the log-logistic and inverse Rayleigh distributions [15]. Rao proposed GASP for gamma distribution by using two points on the OC curve, then developed GASP's for the exponentiated exponential distribution and Marshall Olkin extended Lomax distribution [16,17]. Most of the acceptance sampling plans (ASPs) is designed under the truncated life test based on the mean lifetime. However, the percentile of lifetime is needed in designing ASPs to meet engineering design purpose in many applications. Some literature mentioned the importance to develop ASPs for the product based on percentiles of lifetime instead of the mean lifetime for the different distributions [18-22].

Resubmitted sampling plan consists of repeat sampling process, once the consumer is not accepted a production lot based on a single acceptance sampling plan then take the second sample of same size for testing; and build a decision removing the first sample results under the provisions of the statutes or contract. Therefore, if a lot is rejected on first inspection by a single sampling plan, there submitted sampling plan provides the opportunity to recheck or test the rejected product or lot for the producer and then resubmit the rejected product or lot again. Often, the producer has the empirical information about the product and he may like that this re-sampling process. The resubmitted sampling plan can be applied in many of the situations. For example, a category of "suspect" in the ante-mortem inspection of animals is created for resampling. These resubmitted sampling plans were introduced elsewhere [23]. Recently a group acceptance sampling plan (GASP) for a resubmitting lot when the lifetime of a product follows the Type II Generalized half-logistic distribution (GHLD) is proposed [24-36]. The parameters of the proposed plan such as minimum group size and acceptance number are determined for a pre-specified consumer's risk, number of testers and test termination time. The objective of this paper is to construct GASPs for resubmitted lots under Type II GHLD. In Section 2, we develop a GASP for the resubmitting lot when the lifetime of a product follows Type II GHLD. Section 3 is devoted for presentation of the proposed plan applicable to the industry. In Section 4, the proposed GASP for the resubmitted lot is compared with the ordinary GASP. Finally, the results are demonstrated with a live data set in Section 5. 


\section{Design of Group Acceptance Sampling Plan for Resubmitted Lots}

In this section, according to literature [23] the assumptions made for resubmitted group acceptance sampling plan are as follows:

a) Abiding by the provisions of statute or contract, the information of the original inspection resulting in non-acceptance is required to discard.

b) Producer is honest and consumer has confidence in producer who will not deliberately take the advantage of re-sampling.

The operating procedure of the proposed GASP under a truncated life test for resubmitted lots is carried out as per the following steps:

Step 1: Use the actual group sampling inspection by randomly selecting $n$ items and divide them into $g$ groups in such a way that each group consists of $\mathrm{r}$ items and $n=r g$. Fix upon about the acceptance number $c$, and the experiment time period, $t_{0}$. If the number of failures within $t_{0}$ from all $\mathrm{g}$ groups is not more than $\mathrm{c}$, then the lot is accepted, otherwise it is not accepted. Truncate the experiment and reject the lot if more than $\mathrm{c}$ failures observed in any group.

Step 2: On non-acceptance of the original GASP, apply the proposed plan $w$ times and reject the lot if it is not accepted on $(w-1)^{\text {th }}$ resubmission.

In this paper, we discussed proposed a GASP for a resubmitted lots when the lifetime of the product follows the Type II Generalized half-logistic distribution proposed by Kantam et al. [37]. The cumulative distribution function (c.d.f.) of Type II GHLD is given by

$$
F(t ; \theta, \sigma)=1-\left[\frac{2}{1+e^{t / \sigma}}\right]^{\theta} ; \mathrm{t}>0 \text { and } \sigma, \theta>0
$$

Where $\sigma$ is a scale parameter, $\theta$ is a shape parameter. When $\theta=1$, the Type II GHLD converts to half logistic distribution.

The 100q-th percentile of Type II Generalized half-logistic distribution is given as:

$$
t_{q}=\sigma \eta_{q}, \quad \text { where } \eta_{q}=\ln \left(2(1-q)^{-1 / \theta}-1\right)
$$

The lifetime is $25^{\text {th }}$ percentile of Type II GHLD and is given by

$$
t_{q}=\sigma\left[\ln \left(2(1-0.25)^{-1 / \theta}-1\right)\right]
$$

By assuming the parameter $\theta$ is known, the $25^{\text {th }}$ percentile lifetime given in Equation (3) is the function of scale parameter $\sigma$ only.

Then the probability of accepting lot based on the number of failures from all groups is for the ordinary group sampling plan based on the number of failures from all groups is as given by

$$
L(p)=\sum_{i=0}^{c}\left(\begin{array}{l}
r g \\
i
\end{array}\right) p^{i}(1-p)^{r g-i}
$$

Where $p$ is probability of obtain a failure any group during test termination time, $t_{0}, r$ is the group size, $g$ is the number of testers and $c$ is the acceptance number. Then the lot 
acceptance probability for Type II GHLD, then $p=F\left(t_{0}, \sigma, \theta\right)$. Commonly, it would be easiest to find the test termination time, $t_{0}$, as $t_{0}=\delta_{q} t_{q}^{0}$ for a constant $\delta_{q}$ and the targeted 100q-th percentile lifetime, $t_{q}^{0}$. Then, we can express $p$ as

$$
p=1-\frac{2^{\theta}}{\left[1+\exp \left(\frac{\eta_{q} \delta_{q}^{0}}{\left(t_{q} / t_{q}^{0}\right)}\right)\right]^{\theta}}
$$

Then, the lot acceptance probability in group acceptance sampling plan for the resubmitted lots with (w-1) resubmissions schema will be [23]

$$
P_{a}(p)=1-(1-L(p))^{w} .
$$

Therefore, the lot acceptance probability proposed plan can be given by

$$
P_{a}(p)=1-\left(1-\left\{\sum_{i=0}^{c}\left(\begin{array}{l}
r g \\
i
\end{array}\right) p^{i}(1-p)^{r g-i}\right\}\right)^{w} .
$$

Hence, $c$ and $g$ are two parameters of the in this proposed group acceptance sampling plan for the resubmitted lot, $\mathrm{r}$ is a group size and the pre-specified truncated life test time schedule, $t_{0}=\delta_{q} t_{q}^{0}$, in terms of a multiple of targeted percentile lifetime, $t_{q}^{0}$. When the true 100q-th life time percentile, $t_{q}$, is greater than or equal to the target one, the product is acceptable lot; otherwise, it is not applicable lot. Both consumer and producer want an acceptance sampling plan to make decision satisfy their specified assumption. Consumer want probability of lot acceptance less than at the lot tolerance reliability level (LTRL) and producer requires probability of lot acceptance should be at least $1-\alpha$ at the acceptance reliability level (ARL). Let $p_{1}$ be probability of a failure corresponding to the producer's risk $(\alpha)$ at acceptance reliability level, say of $t_{0} / t_{q}^{0}=2,4,6,8$ in equation (5). On other hand, let $p_{2}$ be probability of a failure corresponding to the consumer's risk $(\beta)^{\text {at }}$ tolerance reliability level, say $t_{0} / t_{q}^{0}=1$, in equation (5). Hence, in this proposed group sampling plan parameters $\mathrm{c}$ and $\mathrm{g}$ can be determined by solving the following inequalities concurrently.

$$
1-\left(1-\left\{\sum_{i=0}^{c}\left(\begin{array}{l}
r g \\
i
\end{array}\right) p_{1}^{i}\left(1-p_{1}\right)^{r g-i}\right\}\right)^{w} \geq 1-\alpha
$$

and

$$
1-\left(1-\left\{\sum_{i=0}^{c}\left(\begin{array}{l}
r g \\
i
\end{array}\right) p_{2}^{i}\left(1-p_{2}\right)^{r g-i}\right\}\right)^{w} \leq \beta
$$


where $p_{1}$ and $p_{2}$ are given by

$$
p_{1}=1-\frac{2^{\theta}}{\left[1+\exp \left(\frac{\eta_{q} \delta_{q}^{0}}{\left(t_{q} / t_{q}^{0}\right)}\right)\right]^{\theta}} \text { and } p_{2}=1-\frac{2^{\theta}}{\left[1+\exp \left(\eta_{q} \delta_{q}^{0}\right)\right]^{\theta}}
$$

Given the producer's risk $\alpha=0.05$ and termination time schedule $t_{0}=\delta_{q} t_{q}^{0}$ with $\delta_{q}^{0}=0.5$ or 1.0, they are two parameters $c$ and $g$ in this proposed group acceptance sampling plan for the resubmitted under the truncated life test at the pre-specified time, $t_{0}$, for the resubmitted lot with $w=2,3$ are obtained according to the consumer's confidence levels $\beta=0.25,0.10,0.05$ and 0.01 . For $w=2$ and the Type II GHLD parameters $\theta=1.5$ the proposed plan parameters are presented in Table 1 for 25th percentile and Table 2 for $10^{\text {th }}$ percentile. For a given $\beta, t_{0} / t_{q}^{0}$, and $r$ at the same respective levels, Table 1 denotes that the number of groups $g$ needed for the proposed sampling plan is decreasing and the acceptance number $c$, is equal (or) increasing as $\delta_{q}$ increases from 0.5 to 1.0. Similarly group size decreases when $r$ increasing from 5 to 1.0. But Table 2 denotes that $g$ is decreasing and $c$ is equal as $\delta_{q}$ increases from 0.5 to 1.0 . The proposed parameters $\theta=2.0$ are displayed in Table 3 for $w=2$, at $25^{\text {th }}$ percentile and Table 4 for $w=2$, at $10^{\text {th }}$ percentile. Table 3 is showing the aforesaid as Table 1, and Table 4 is showing the $g$ is decreasing and $c$ is equal as $\delta_{q}$ increases from 0.5 to 1.0. Tables 5 and 6 are constructed for $w=2$, at $25^{\text {th }}$ percentile and $10^{\text {th }}$ percentile using the MLEs $\hat{\theta}=0.6809$. The proposed plan parameters $\theta=1.5$ are displayed in Table 7 for $w=3$, at $25^{\text {th }}$ percentile and Table 8 for $w=3$, at $10^{\text {th }}$ percentile. The proposed plan parameters $\theta=2.0$ are presented in Table 9 for $w=3$, at $25^{\text {th }}$ percentile and Table 10 for $w=3$, at $10^{\text {th }}$ percentile. Tables 11 and 12 are constructed for $w=3$, at $25^{\text {th }}$ percentile and $10^{\text {th }}$ percentile using the MLEs $\hat{\theta}=0.6809$. For $w=3$ and the Type II GHLD parameters $\theta=1.5$ the sampling parameters are presented in Tables 7 for $25^{\text {th }}$ percentile lifetime and Table 8 for $10^{\text {th }}$ percentile lifetime. For $w=3$ and the Type II GHLD parameters $\theta=2.0$ the sampling parameters are displayed in Table 9 for $25^{\text {th }}$ percentile lifetime and Table 10 for $10^{\text {th }}$ percentile lifetime. Also for $w=3$ and the Type II GHLD estimated parameters $\theta=0.6809$ the sampling parameters are presented in Table 11 for $25^{\text {th }}$ percentile lifetime and Table 12 for $10^{\text {th }}$ percentile lifetime. 
Table 1. Proposed plan having $w=2$ under Type II GHLD for $25^{\text {th }}$ percentile for $\theta=1.5$.

\begin{tabular}{|c|c|c|c|c|c|c|c|c|c|c|c|c|c|}
\hline \multirow{3}{*}{$\beta$} & \multirow{3}{*}{$t_{q} / t_{q}^{0}$} & \multicolumn{6}{|c|}{$r=5$} & \multicolumn{6}{|c|}{$r=10$} \\
\hline & & \multicolumn{3}{|c|}{$\delta_{q}^{0}=0.5$} & \multicolumn{3}{|c|}{$\delta_{q}^{0}=1.0$} & \multicolumn{3}{|c|}{$\delta_{q}^{\mathrm{o}}=0.5$} & \multicolumn{3}{|c|}{$\delta_{q}^{0}=1.0$} \\
\hline & & $C$ & $G$ & pa & $c$ & $\mathrm{~g}$ & pa & $c$ & $\mathrm{~g}$ & pa & C & G & $\mathrm{Pa}$ \\
\hline \multirow{4}{*}{0.25} & 2 & 7 & 17 & 0.9634 & 7 & 9 & 0.9525 & 8 & 10 & 0.9574 & - & - & - \\
\hline & 4 & 2 & 8 & 0.9794 & 2 & 4 & 0.9807 & 2 & 4 & 0.9794 & 5 & 6 & 0.9612 \\
\hline & 6 & 1 & 6 & 0.9803 & 1 & 3 & 0.981 & 1 & 3 & 0.9803 & 1 & 2 & 0.9528 \\
\hline & 8 & 0 & 3 & 0.9513 & 1 & 3 & 0.9927 & 1 & 3 & 0.9924 & 1 & 2 & 0.9806 \\
\hline \multirow{4}{*}{0.10} & 2 & 11 & 28 & 0.9579 & 10 & 13 & 0.9554 & 11 & 14 & 0.9579 & - & - & - \\
\hline & 4 & 3 & 12 & 0.9818 & 2 & 5 & 0.9507 & 3 & 6 & 0.9818 & 5 & 6 & 0.9612 \\
\hline & 6 & 1 & 7 & 0.968 & 1 & 4 & 0.9528 & 1 & 4 & 0.952 & 1 & 2 & 0.9528 \\
\hline & 8 & 1 & 7 & 0.9872 & 1 & 4 & 0.9806 & 1 & 4 & 0.9802 & 1 & 2 & 0.9806 \\
\hline \multirow{4}{*}{0.05} & 2 & 13 & 34 & 0.9504 & 13 & 17 & 0.9593 & 13 & 17 & 0.9 .504 & - & - & - \\
\hline & 4 & 3 & 13 & 0.9726 & 3 & 7 & 0.9628 & 3 & 7 & 0.9606 & 5 & 6 & 0.9612 \\
\hline & 6 & 2 & 11 & 0.9855 & 1 & 4 & 0.9528 & 2 & 6 & 0.9789 & 1 & 2 & 0.9528 \\
\hline & 8 & 1 & 9 & 0.9712 & 1 & 4 & 0.9806 & 1 & 5 & 0.9602 & 1 & 2 & 0.9806 \\
\hline \multirow{4}{*}{0.01} & 2 & 20 & 53 & 0.9558 & 20 & 27 & 0.9558 & 21 & 28 & 0.9538 & - & - & - \\
\hline & 4 & 4 & 19 & 0.9587 & 5 & 11 & 0.978 & 5 & 11 & 0.976 & 5 & 6 & 0.9612 \\
\hline & 6 & 2 & 14 & 0.9603 & 2 & 7 & 0.9615 & 2 & 7 & 0.9603 & 3 & 4 & 0.9907 \\
\hline & 8 & 2 & 14 & 0.9881 & 2 & 7 & 0.9886 & 2 & 7 & 0.9881 & 2 & 4 & 09794 \\
\hline
\end{tabular}

Table 2. Proposed plan having $w=2$ under Type II GHLD for $10^{\text {th }}$ percentile for $\theta=1.5$.

\begin{tabular}{|c|c|c|c|c|c|c|c|c|c|c|c|c|c|}
\hline \multirow{3}{*}{$\beta$} & \multirow{3}{*}{$t_{q} / t_{q}^{0}$} & \multicolumn{6}{|c|}{$r=5$} & \multicolumn{6}{|c|}{$r=10$} \\
\hline & & \multicolumn{3}{|c|}{$\delta_{q}^{0}=0.5$} & \multicolumn{3}{|c|}{$\delta_{q}^{0}=1.0$} & \multicolumn{3}{|c|}{$\delta_{q}^{0}=0.5$} & \multicolumn{3}{|c|}{$\delta_{q}^{0}=1.0$} \\
\hline & & $C$ & G & pa & $c$ & $\mathrm{~g}$ & $\mathrm{pa}$ & $c$ & $\mathrm{~g}$ & $\mathrm{~Pa}$ & $C$ & G & $\mathrm{Pa}$ \\
\hline \multirow{4}{*}{0.25} & 2 & 7 & 44 & 0.9607 & 7 & 22 & 0.9629 & 7 & 22 & 0.9607 & 7 & 11 & 0.9629 \\
\hline & 4 & 1 & 14 & 0.950 & 1 & 7 & 0.9505 & 1 & 7 & 0.95 & 2 & 5 & 0.9822 \\
\hline & 6 & 1 & 14 & 0.9858 & 1 & 7 & 0.986 & 1 & 7 & 0.9858 & 1 & 4 & 0.9784 \\
\hline & 8 & 1 & 14 & 0.9946 & 1 & 7 & 0.9947 & 1 & 7 & 0.9946 & 1 & 4 & 0.9916 \\
\hline \multirow{4}{*}{0.10} & 2 & 11 & 72 & 0.9569 & 11 & 36 & 0.9597 & 11 & 36 & 0.9569 & 11 & 18 & 0.9597 \\
\hline & 4 & 2 & 25 & 0.9546 & 3 & 15 & 0.9848 & 2 & 16 & 0.9781 & 3 & 8 & 0.9787 \\
\hline & 6 & 1 & 19 & 0.9628 & 1 & 10 & 0.9569 & 1 & 10 & 0.9566 & 1 & 5 & 0.9569 \\
\hline & 8 & 1 & 19 & 0.9849 & 1 & 10 & 0.9823 & 1 & 10 & 0.9822 & 1 & 5 & 0.9823 \\
\hline \multirow{4}{*}{0.05} & 2 & 14 & 92 & 0.9615 & 4 & 4 & 0.9643 & 14 & 46 & 0.9615 & 14 & 23 & 0.9643 \\
\hline & 4 & 3 & 35 & 0.9660 & 2 & 2 & 0.9866 & 3 & 18 & 0.9611 & 3 & 9 & 0.9619 \\
\hline & 6 & 2 & 29 & 0.9841 & 2 & 2 & 0.9964 & 2 & 15 & 0.9816 & 2 & 7 & 0.9866 \\
\hline & 8 & 1 & 22 & 0.9758 & 1 & 1 & 0.976 & 1 & 11 & 0.9758 & 1 & 6 & 0.9686 \\
\hline \multirow{4}{*}{0.01} & 2 & 20 & 136 & 0.9561 & 20 & 20 & 0.9596 & 20 & 68 & 0.9561 & 20 & 34 & 0.9566 \\
\hline & 4 & 4 & 49 & 0.9587 & 4 & 4 & 0.9549 & 4 & 25 & 0.954 & 15 & 14 & 0.9785 \\
\hline & 6 & 2 & 36 & 0.9611 & 2 & 2 & 0.9615 & 2 & 18 & 0.9611 & 2 & 9 & 0.9615 \\
\hline & 8 & 2 & 36 & 0.9883 & 2 & 2 & 0.9885 & 2 & 18 & 0.9883 & 2 & 9 & 0.9885 \\
\hline
\end{tabular}


Table 3. Proposed plan having $w=2$ under Type II GHLD for $25^{\text {th }}$ percentile for $\theta=2.0$.

\begin{tabular}{|c|c|c|c|c|c|c|c|c|c|c|c|c|c|}
\hline \multirow{3}{*}{$\beta$} & \multirow{3}{*}{$t_{q} / t_{q}^{0}$} & \multicolumn{6}{|c|}{$r=5$} & \multicolumn{6}{|c|}{$r=10$} \\
\hline & & \multicolumn{3}{|c|}{$\delta_{q}^{0}=0.5$} & \multicolumn{3}{|c|}{$\delta_{q}^{0}=1.0$} & \multicolumn{3}{|c|}{$\delta_{q}^{0}=0.5$} & \multicolumn{3}{|c|}{$\delta_{q}^{0}=1.0$} \\
\hline & & $C$ & $\mathrm{G}$ & $\mathrm{pa}$ & $c$ & $\mathrm{~g}$ & pa & $c$ & $\mathrm{~g}$ & pa & $C$ & $\mathrm{G}$ & $\mathrm{Pa}$ \\
\hline \multirow{4}{*}{0.25} & 2 & 7 & 17 & 0.9597 & 8 & 10 & 0.9617 & 8 & 10 & 0.9527 & - & - & - \\
\hline & 4 & 2 & 8 & 0.9779 & 2 & 4 & 0.9796 & 2 & 4 & 0.9779 & 5 & 6 & 0.9579 \\
\hline & 6 & 1 & 6 & 0.9792 & 1 & 3 & 0.98 & 1 & 3 & 0.9792 & 1 & 2 & 0.9506 \\
\hline & 8 & 1 & 6 & 0.9919 & 1 & 3 & 0.9923 & 1 & 3 & 0.9919 & 1 & 2 & 0.9796 \\
\hline \multirow{4}{*}{0.10} & 2 & 11 & 28 & 0.9525 & 10 & 13 & 0.952 & 11 & 14 & 0.9525 & - & - & - \\
\hline & 4 & 3 & 12 & 0.9802 & 3 & 6 & 0.982 & 3 & 6 & 0.9802 & 5 & 6 & 0.9579 \\
\hline & 6 & 1 & 7 & 0.9662 & 1 & 4 & 0.9506 & 2 & 5 & 0.9898 & 1 & 2 & 0.9506 \\
\hline & 8 & 1 & 7 & 0.9864 & 1 & 4 & 0.9796 & 1 & 4 & 0.9790 & 1 & 2 & 0.9796 \\
\hline \multirow{4}{*}{0.05} & 2 & 14 & 36 & 0.954 & 13 & 17 & 0.9556 & 14 & 18 & 0.9540 & - & - & - \\
\hline & 4 & 3 & 13 & 0.9703 & 3 & 7 & 0.9602 & 3 & 7 & 0.9575 & 5 & 6 & 0.9579 \\
\hline & 6 & 2 & 11 & 0.9844 & 1 & 4 & 0.9506 & 2 & 6 & 0.9774 & 1 & 2 & 0.9506 \\
\hline & 8 & 1 & 9 & 0.9696 & 1 & 4 & 0.9796 & 1 & 5 & 0.9581 & 1 & 2 & 0.9796 \\
\hline \multirow{4}{*}{0.01} & 2 & 19 & 50 & 0.9506 & 20 & 27 & 0.9509 & 19 & 25 & 0.9506 & - & - & - \\
\hline & 4 & 4 & 19 & 0.955 & 5 & 11 & 0.9759 & 5 & 11 & 0.9734 & 5 & 6 & 0.9579 \\
\hline & 6 & 2 & 14 & 0.9576 & 2 & 7 & 0.9591 & 2 & 7 & 0.9576 & 3 & 4 & 0.9899 \\
\hline & 8 & 2 & 14 & 0.9871 & 2 & 7 & 0.9877 & 2 & 7 & 0.9871 & 2 & 4 & 0.9779 \\
\hline
\end{tabular}

Table 4. Proposed plan having $w=2$ under Type II GHLD for $10^{\text {th }}$ percentile for $\theta=2.0$.

\begin{tabular}{|c|c|c|c|c|c|c|c|c|c|c|c|c|c|}
\hline \multirow{3}{*}{$\beta$} & \multirow{3}{*}{$t_{q} / t_{q}^{0}$} & \multicolumn{6}{|c|}{$r=5$} & \multicolumn{6}{|c|}{$r=10$} \\
\hline & & \multicolumn{3}{|c|}{$\delta_{q}^{0}=0.5$} & \multicolumn{3}{|c|}{$\delta_{q}^{0}=1.0$} & \multicolumn{3}{|c|}{$\delta_{q}^{0}=0.5$} & \multicolumn{3}{|c|}{$\delta_{q}^{0}=1.0$} \\
\hline & & $C$ & $G$ & $\mathrm{pa}$ & $c$ & $\mathrm{~g}$ & $\mathrm{pa}$ & $c$ & $\mathrm{~g}$ & $\mathrm{pa}$ & $C$ & $\mathrm{G}$ & $\mathrm{Pa}$ \\
\hline \multirow{4}{*}{0.25} & 2 & 7 & 44 & 0.959 & 7 & 22 & 0.9619 & 7 & 22 & 0.959 & 7 & 11 & 0.9619 \\
\hline & 4 & 2 & 20 & 0.9812 & 2 & 10 & 0.9817 & 2 & 10 & 0.9812 & 2 & 5 & 0.9817 \\
\hline & 6 & 1 & 14 & 0.9854 & 1 & 7 & 0.9857 & 1 & 7 & 0.9854 & 1 & 4 & 0.9779 \\
\hline & 8 & 1 & 14 & 0.9944 & 1 & 7 & 0.9945 & 1 & 7 & 0.9944 & 1 & 4 & 0.9914 \\
\hline \multirow{4}{*}{0.10} & 2 & 11 & 71 & 0.9599 & 11 & 36 & 0.9583 & 11 & 36 & 0.9547 & 11 & 18 & 0.9583 \\
\hline & 4 & 2 & 25 & 0.9534 & 3 & 15 & 0.943 & 3 & 15 & 0.9837 & 3 & 8 & 0.9781 \\
\hline & 6 & 1 & 19 & 0.962 & 1 & 10 & 0.956 & 1 & 10 & 0.9557 & 1 & 5 & 0.956 \\
\hline & 8 & 1 & 19 & 0.9845 & 1 & 10 & 0.981 & 1 & 10 & 0.9897 & 1 & 5 & 0.9819 \\
\hline \multirow{4}{*}{0.05} & 2 & 14 & 92 & 0.9591 & 14 & 46 & 0.9628 & 14 & 46 & 0.9591 & 14 & 23 & 0.9628 \\
\hline & 4 & 3 & 34 & 0.9694 & 3 & 17 & 0.9703 & 3 & 17 & 0.9694 & 3 & 9 & 0.9608 \\
\hline & 6 & 2 & 28 & 0.9859 & 2 & 14 & 0.6862 & 2 & 14 & 0.9859 & 2 & 7 & 0.9862 \\
\hline & 8 & 1 & 22 & 0.9753 & 1 & 11 & 0.9755 & 1 & 11 & 0.9753 & 1 & 6 & 0.9679 \\
\hline \multirow{4}{*}{0.01} & 2 & 20 & 136 & 0.9529 & 20 & 68 & 0.9576 & 20 & 68 & 0.9529 & 20 & 34 & 0.9576 \\
\hline & 4 & 4 & 49 & 0.9571 & 4 & 25 & 0.9535 & 4 & 25 & 0.9523 & 5 & 14 & 0.9776 \\
\hline & 6 & 2 & 36 & 0.9599 & 2 & 18 & 0.9605 & 2 & 18 & 0.9599 & 2 & 9 & 0.9605 \\
\hline & 8 & 2 & 36 & 0.9879 & 2 & 18 & 0.9881 & 2 & 18 & 0.9879 & 2 & 9 & 0.9881 \\
\hline
\end{tabular}


Table 5. Proposed plan having $w=2$ under Type II GHLD for $25^{\text {th }}$ percentile for $\hat{\theta}=0.6809$.

\begin{tabular}{|c|c|c|c|c|c|c|c|c|c|c|c|c|c|}
\hline \multirow{3}{*}{$\beta$} & \multirow{3}{*}{$t_{q} / t_{q}^{0}$} & \multicolumn{6}{|c|}{$r=5$} & \multicolumn{6}{|c|}{$r=10$} \\
\hline & & \multicolumn{3}{|c|}{$\delta_{q}^{0}=0.5$} & \multicolumn{3}{|c|}{$\delta_{q}^{\mathrm{O}}=1.0$} & \multicolumn{3}{|c|}{$\delta_{q}^{0}=0.5$} & \multicolumn{3}{|c|}{$\delta_{q}^{\mathrm{O}}=1.0$} \\
\hline & & $C$ & $\mathrm{G}$ & pa & $c$ & $\mathrm{~g}$ & $\mathrm{~Pa}$ & $c$ & $\mathrm{~g}$ & pa & $C$ & $\mathrm{G}$ & $\mathrm{Pa}$ \\
\hline \multirow{4}{*}{0.25} & 2 & 7 & 18 & 0.9625 & 7 & 9 & 0.9629 & 7 & 9 & 0.9625 & - & - & - \\
\hline & 4 & 2 & 8 & 0.9845 & 2 & 4 & 0.9848 & 2 & 4 & 0.9845 & 4 & 5 & 0.9616 \\
\hline & 6 & 1 & 6 & 0.9843 & 1 & 3 & 0.9844 & 1 & 3 & 0.9843 & 1 & 2 & 0.9608 \\
\hline & 8 & 1 & 6 & 0.9940 & 1 & 3 & 0.9942 & 1 & 3 & 0.9940 & 1 & 2 & 0.9844 \\
\hline \multirow{4}{*}{0.10} & 2 & 10 & 27 & 0.9536 & 9 & 12 & 0.9559 & 11 & 15 & 0.9517 & - & - & - \\
\hline & 4 & 2 & 10 & 0.9603 & 2 & 5 & 0.9603 & 2 & 5 & 0.9603 & 4 & 5 & 0.9616 \\
\hline & 6 & 1 & 8 & 0.9610 & 1 & 4 & 0.9608 & 1 & 4 & 0.9610 & 1 & 2 & 0.9608 \\
\hline & 8 & 1 & 8 & 0.9843 & 1 & 4 & 0.9844 & 1 & 4 & 0.9843 & 1 & 2 & 0.9844 \\
\hline \multirow{4}{*}{0.05} & 2 & 12 & 33 & 0.9501 & 11 & 15 & 0.9511 & 14 & 19 & 0.9598 & - & - & - \\
\hline & 4 & 3 & 14 & 0.9714 & 3 & 7 & 0.9715 & 3 & 7 & 0.9714 & 4 & 5 & 0.9616 \\
\hline & 6 & 2 & 12 & 0.9844 & 1 & 4 & 0.9608 & 2 & 6 & 0.9844 & 1 & 2 & 0.9608 \\
\hline & 8 & 1 & 9 & 0.9770 & 1 & 4 & 0.9844 & 1 & 5 & 0.9680 & 1 & 2 & 0.9844 \\
\hline \multirow{4}{*}{0.01} & 2 & 18 & 50 & 0.9561 & 18 & 25 & 0.9551 & 14 & 25 & 0.9561 & - & - & - \\
\hline & 4 & 4 & 20 & 0.9618 & 4 & 10 & 0.9616 & 3 & 10 & 0.9618 & 4 & 5 & 0.9616 \\
\hline & 6 & 2 & 15 & 0.9604 & 2 & 7 & 0.9700 & 2 & 9 & 0.9871 & 3 & 4 & 0.9934 \\
\hline & 8 & 2 & 15 & 0.9882 & 2 & 7 & 0.9915 & 1 & 8 & 0.9843 & 2 & 4 & 0.9845 \\
\hline
\end{tabular}

Table 6. Proposed plan having $w=2$ under Type II GHLD for $10^{\text {th }}$ percentile for $\hat{\theta}=0.6809$.

\begin{tabular}{|c|c|c|c|c|c|c|c|c|c|c|c|c|c|}
\hline \multirow{3}{*}{$\beta$} & \multirow{3}{*}{$t_{q} / t_{q}^{0}$} & \multicolumn{6}{|c|}{$r=5$} & \multicolumn{6}{|c|}{$r=10$} \\
\hline & & \multicolumn{3}{|c|}{$\delta_{q}^{0}=0.5$} & \multicolumn{3}{|c|}{$\delta_{q}^{\mathrm{o}}=1.0$} & \multicolumn{3}{|c|}{$\delta_{q}^{0}=0.5$} & \multicolumn{3}{|c|}{$\delta_{q}^{0}=1.0$} \\
\hline & & $C$ & $\mathrm{G}$ & pa & $c$ & $\mathrm{~g}$ & $\mathrm{~Pa}$ & $c$ & $\mathrm{~g}$ & $\mathrm{~Pa}$ & $C$ & $\mathrm{G}$ & $\mathrm{Pa}$ \\
\hline \multirow{4}{*}{0.25} & 2 & 7 & 45 & 0.9618 & 7 & 22 & 0.9673 & 7 & 23 & 0.9553 & 11 & 7 & 0.9673 \\
\hline & 4 & 1 & 14 & 0.9544 & 1 & 7 & 0.9542 & 1 & 7 & 0.9544 & 2 & 5 & 0.9841 \\
\hline & 6 & 1 & 14 & 0.9872 & 1 & 7 & 0.9873 & 1 & 7 & 0.9872 & 1 & 4 & 0.9803 \\
\hline & 8 & 0 & 8 & 0.9520 & 0 & 4 & 0.9518 & 0 & 4 & 0.9522 & 1 & 4 & 0.9924 \\
\hline \multirow{4}{*}{0.10} & 2 & 10 & 68 & 0.9500 & 11 & 36 & 0.9655 & 10 & 34 & 0.9500 & 11 & 18 & 0.9655 \\
\hline & 4 & 2 & 25 & 0.9598 & 2 & 13 & 0.9530 & 2 & 13 & 0.9532 & 3 & 8 & 0.9814 \\
\hline & 6 & 1 & 19 & 0.9663 & 1 & 10 & 0.9605 & 1 & 10 & 0.9606 & 1 & 5 & 0.9605 \\
\hline & 8 & 1 & 19 & 0.9865 & 1 & 10 & 0.9840 & 1 & 10 & 0.9840 & 1 & 5 & 0.9840 \\
\hline \multirow{4}{*}{0.05} & 2 & 13 & 89 & 0.9534 & 12 & 41 & 0.9512 & 14 & 47 & 0.9633 & 13 & 22 & 0.9573 \\
\hline & 4 & 3 & 35 & 0.9707 & 3 & 17 & 0.9747 & 3 & 18 & 0.9664 & 3 & 9 & 0.9664 \\
\hline & 6 & 2 & 29 & 0.9862 & 2 & 14 & 0.9882 & 2 & 15 & 0.9840 & 2 & 7 & 0.9882 \\
\hline & 8 & 1 & 22 & 0.9783 & 1 & 11 & 0.9783 & 1 & 11 & 0.9783 & 1 & 6 & 0.9714 \\
\hline \multirow{4}{*}{0.01} & 2 & 20 & 139 & 0.9584 & 18 & 63 & 0.9501 & 20 & 70 & 0.9747 & 19 & 33 & 0.9558 \\
\hline & 4 & 4 & 50 & 0.9610 & 4 & 25 & 0.9609 & 4 & 25 & 0.9610 & 4 & 13 & 0.9517 \\
\hline & 6 & 2 & 37 & 0.9619 & 2 & 18 & 0.9658 & 2 & 19 & 0.9577 & 2 & 9 & 0.9658 \\
\hline & 8 & 2 & 37 & 0.9886 & 2 & 18 & 0.9900 & 2 & 19 & 0.9872 & 2 & 9 & 0.9900 \\
\hline
\end{tabular}


Table 7. Proposed plan having $w=3$ under Type II GHLD for $25^{\text {th }}$ percentile for $\theta=1.5$.

\begin{tabular}{|c|c|c|c|c|c|c|c|c|c|c|c|c|c|}
\hline \multirow{3}{*}{$\beta$} & \multirow{3}{*}{$t_{q} / t_{q}^{0}$} & \multicolumn{6}{|c|}{$r=5$} & \multicolumn{6}{|c|}{$r=10$} \\
\hline & & \multicolumn{3}{|c|}{$\delta_{q}^{0}=0.5$} & \multicolumn{3}{|c|}{$\delta_{q}^{\mathrm{o}}=1.0$} & \multicolumn{3}{|c|}{$\delta_{q}^{0}=0.5$} & \multicolumn{3}{|c|}{$\delta_{q}^{0}=1.0$} \\
\hline & & $C$ & $\mathrm{G}$ & pa & $c$ & $\mathrm{~g}$ & pa & $c$ & $\mathrm{~g}$ & pa & $C$ & $\mathrm{G}$ & pa \\
\hline \multirow{4}{*}{0.25} & 2 & 5 & 15 & 0.951 & 6 & 9 & 0.9535 & 10 & 7 & 0.9641 & - & - & - \\
\hline & 4 & 1 & 6 & 0.9826 & 1 & 3 & 0.9831 & 1 & 3 & 0.9826 & 2 & 3 & 0.9696 \\
\hline & 6 & 1 & 4 & 0.9539 & 0 & 2 & 0.9531 & 0 & 2 & 0.9539 & 0 & 1 & 0.9531 \\
\hline & 8 & 1 & 4 & 0.9774 & 0 & 2 & 0.9771 & 0 & 2 & 0.9774 & 0 & 1 & 0.9771 \\
\hline \multirow{4}{*}{0.10} & 2 & 8 & 23 & 0.9615 & 8 & 12 & 0.9502 & 9 & 13 & 0.9593 & - & - & - \\
\hline & 4 & 2 & 11 & 0.9802 & 2 & 5 & 0.9891 & 2 & 6 & 0.9685 & 2 & 3 & 0.9696 \\
\hline & 6 & 1 & 8 & 0.9895 & 1 & 4 & 0.9897 & 1 & 4 & 0.9895 & 1 & 2 & 0.9897 \\
\hline & 8 & 0 & 5 & 0.9607 & 1 & 4 & 0.9973 & 1 & 4 & 0.9972 & 1 & 2 & 0.9973 \\
\hline \multirow{4}{*}{0.05} & 2 & 10 & 29 & 0.9574 & 11 & 16 & 0.9628 & 11 & 16 & 0.9557 & - & - & - \\
\hline & 4 & 2 & 12 & 0.9685 & 2 & 6 & 0.9696 & 2 & 6 & 0.9685 & 2 & 3 & 0.9696 \\
\hline & 6 & 1 & 9 & 0.9825 & 1 & 5 & 0.9732 & 1 & 5 & 0.9728 & 2 & 3 & 0.9971 \\
\hline & 8 & 1 & 9 & 0.9951 & 1 & 5 & 0.9922 & 1 & 5 & 0.9929 & 1 & 3 & 0.9826 \\
\hline \multirow{4}{*}{0.01} & 2 & 15 & 44 & 0.9509 & 15 & 22 & 0.9596 & 15 & 22 & 0.9509 & - & - & - \\
\hline & 4 & 3 & 17 & 0.9714 & 3 & 9 & 0.9611 & 3 & 9 & 0.9595 & 4 & 5 & 0.9883 \\
\hline & 6 & 2 & 15 & 0.9882 & 2 & 7 & 0.9924 & 2 & 8 & 0.983 & 2 & 4 & 0.9835 \\
\hline & 8 & 1 & 12 & 0.9824 & 1 & 6 & 0.9826 & 1 & 6 & 0.9824 & 1 & 3 & 0.9826 \\
\hline
\end{tabular}

Table 8. Proposed plan having $w=3$ under Type II GHLD for $10^{\text {th }}$ percentile for $\theta=1.5$.

\begin{tabular}{|c|c|c|c|c|c|c|c|c|c|c|c|c|c|}
\hline \multirow{3}{*}{$\beta$} & \multirow{3}{*}{$t_{q} / t_{q}^{0}$} & \multicolumn{6}{|c|}{$r=5$} & \multicolumn{6}{|c|}{$r=10$} \\
\hline & & \multicolumn{3}{|c|}{$\delta_{q}^{0}=0.5$} & \multicolumn{3}{|c|}{$\delta_{q}^{0}=1.0$} & \multicolumn{3}{|c|}{$\delta_{q}^{\mathrm{O}}=0.5$} & \multicolumn{3}{|c|}{$\delta_{q}^{\mathrm{O}}=1.0$} \\
\hline & & $C$ & $\mathrm{G}$ & pa & $c$ & $\mathrm{G}$ & pa & $c$ & $\mathrm{~g}$ & $\mathrm{~Pa}$ & $C$ & $\mathrm{G}$ & $\mathrm{Pa}$ \\
\hline \multirow{4}{*}{0.25} & 2 & 5 & 37 & 0.9642 & 5 & 19 & 0.958 & 5 & 19 & 0.9564 & 6 & 11 & 0.9670 \\
\hline & 4 & 1 & 16 & 0.9802 & 1 & 8 & 0.9804 & 1 & 8 & 0.9802 & 1 & 4 & 0.9804 \\
\hline & 6 & 0 & 10 & 0.9583 & 0 & 5 & 0.9580 & 0 & 5 & 0.9583 & 1 & 4 & 0.9968 \\
\hline & 8 & 0 & 10 & 0.9796 & 0 & 5 & 0.9795 & 0 & 5 & 0.9796 & 0 & 3 & 0.9676 \\
\hline \multirow{4}{*}{0.10} & 2 & 8 & 60 & 0.9562 & 8 & 13 & 0.9583 & 8 & 30 & 0.9562 & 8 & 15 & 0.9538 \\
\hline & 4 & 2 & 27 & 0.9850 & 1 & 10 & 0.9521 & 2 & 14 & 0.9817 & 1 & 5 & 0.9521 \\
\hline & 6 & 1 & 21 & 0.9888 & 1 & 10 & 0.9911 & 1 & 11 & 0.9863 & 1 & 5 & 0.9911 \\
\hline & 8 & 0 & 13 & 0.9608 & 0 & 7 & 0.9530 & 0 & 7 & 0.9532 & 1 & 5 & 0.9977 \\
\hline \multirow{4}{*}{0.05} & 2 & 10 & 75 & 0.9551 & 10 & 38 & 0.9514 & 11 & 41 & 0.9577 & 10 & 19 & 0.9514 \\
\hline & 4 & 2 & 31 & 0.9687 & 2 & 15 & 0.9639 & 2 & 16 & 0.9632 & 2 & 8 & 0.9636 \\
\hline & 6 & 1 & 24 & 0.9802 & 1 & 12 & 0.9803 & 1 & 12 & 0.9802 & 1 & 6 & 0.9803 \\
\hline & 8 & 1 & 24 & 0.9944 & 1 & 12 & 0.9944 & 1 & 12 & 0.9944 & 1 & 6 & 0.9944 \\
\hline \multirow{4}{*}{0.01} & 2 & 16 & 119 & 0.9594 & 15 & 57 & 0.9501 & 12 & 60 & 0.9549 & 16 & 30 & 0.9578 \\
\hline & 4 & 3 & 45 & 0.9669 & 9 & 23 & 0.9628 & 3 & 23 & 0.9622 & 3 & 12 & 0.9523 \\
\hline & 6 & 2 & 38 & 0.9895 & 2 & 19 & 0.9896 & 2 & 19 & 0.9895 & 2 & 10 & 0.9861 \\
\hline & 8 & 1 & 31 & 0.9826 & 1 & 16 & 0.9802 & 1 & 16 & 0.9801 & 1 & 8 & 0.9802 \\
\hline
\end{tabular}


Table 9. Proposed plan having $w=3$ under Type II GHLD for $25^{\text {th }}$ percentile for $\theta=2.0$.

\begin{tabular}{|c|c|c|c|c|c|c|c|c|c|c|c|c|c|}
\hline \multirow{3}{*}{$\beta$} & \multirow{3}{*}{$t_{q} / t_{q}^{0}$} & \multicolumn{6}{|c|}{$r=5$} & \multicolumn{6}{|c|}{$r=10$} \\
\hline & & \multicolumn{3}{|c|}{$\delta_{q}^{0}=0.5$} & \multicolumn{3}{|c|}{$\delta_{q}^{0}=1.0$} & \multicolumn{3}{|c|}{$\delta_{q}^{0}=0.5$} & \multicolumn{3}{|c|}{$\delta_{q}^{0}=1.0$} \\
\hline & & $C$ & G & pa & $c$ & $\mathrm{~g}$ & $\mathrm{~Pa}$ & $c$ & $\mathrm{~g}$ & pa & $C$ & $G$ & $\mathrm{~Pa}$ \\
\hline \multirow{4}{*}{0.25} & 2 & 6 & 16 & 0.9783 & 6 & 9 & 0.9502 & 6 & 8 & 0.9783 & - & - & - \\
\hline & 4 & 1 & 6 & 0.9814 & 1 & 3 & 0.9821 & 1 & 3 & 0.9814 & 2 & 3 & 0.9674 \\
\hline & 6 & 0 & 4 & 0.952 & 0 & 2 & 0.9513 & 0 & 2 & 0.9520 & 0 & 1 & 0.9513 \\
\hline & 8 & 0 & 4 & 0.9763 & 0 & 2 & 0.9761 & 0 & 2 & 0.9763 & 0 & 1 & 0.9761 \\
\hline \multirow{4}{*}{0.10} & 2 & 8 & 23 & 0.9563 & 9 & 13 & 0.9622 & 9 & 13 & 0.9535 & - & - & - \\
\hline & 4 & 2 & 10 & 0.9873 & 2 & 5 & 0.9882 & 2 & 5 & 0.9873 & 2 & 3 & 0.9674 \\
\hline & 6 & 1 & 8 & 0.9887 & 1 & 4 & 0.9890 & 1 & 4 & 0.9887 & 1 & 2 & 0.9890 \\
\hline & 8 & 0 & 5 & 0.9590 & 1 & 4 & 0.9971 & 1 & 4 & 0.9970 & 1 & 2 & 0.9971 \\
\hline \multirow{4}{*}{0.05} & 2 & 10 & 29 & 0.9511 & 11 & 16 & 0.9590 & 12 & 17 & 0.9617 & - & - & - \\
\hline & 4 & 2 & 12 & 0.9658 & 2 & 6 & 0.9674 & 2 & 6 & 0.9658 & 2 & 3 & 0.9674 \\
\hline & 6 & 1 & 9 & 0.9811 & 1 & 5 & 0.9714 & 1 & 5 & 0.9709 & 2 & 3 & 0.9968 \\
\hline & 8 & 1 & 9 & 0.9947 & 1 & 5 & 0.9917 & 1 & 5 & 0.9914 & 1 & 3 & 0.9814 \\
\hline \multirow{4}{*}{0.01} & 2 & 16 & 46 & 0.9550 & 15 & 22 & 0.9549 & 16 & 23 & 0.9550 & - & - & - \\
\hline & 4 & 3 & 17 & 0.9684 & 3 & 9 & 0.9578 & 3 & 9 & 0.9555 & 4 & 5 & 0.9870 \\
\hline & 6 & 2 & 15 & 0.9870 & 2 & 7 & 0.9917 & 2 & 8 & 0.9813 & 2 & 4 & 0.9821 \\
\hline & 8 & 1 & 12 & 0.9810 & 1 & 6 & 0.9814 & 1 & 6 & 0.9810 & 1 & 1 & 0.9814 \\
\hline
\end{tabular}

Table 10. Proposed plan having $w=3$ under Type II GHLD for $10^{\text {th }}$ percentile for $\theta=2.0$.

\begin{tabular}{|c|c|c|c|c|c|c|c|c|c|c|c|c|c|}
\hline \multirow{3}{*}{$\beta$} & \multirow{3}{*}{$t_{q} / t_{q}^{0}$} & \multicolumn{6}{|c|}{$r=5$} & \multicolumn{6}{|c|}{$r=10$} \\
\hline & & \multicolumn{3}{|c|}{$\delta_{q}^{0}=0.5$} & \multicolumn{3}{|c|}{$\delta_{q}^{0}=1.0$} & \multicolumn{3}{|c|}{$\delta_{q}^{0}=0.5$} & \multicolumn{3}{|c|}{$\delta_{q}^{0}=1.0$} \\
\hline & & $C$ & $\mathrm{G}$ & pa & $c$ & $\mathrm{G}$ & pa & $c$ & $\mathrm{~g}$ & pa & $C$ & $G$ & $\mathrm{~Pa}$ \\
\hline \multirow{4}{*}{0.25} & 2 & 5 & 37 & 0.9626 & 5 & 19 & 0.9568 & 5 & 19 & 0.9545 & 6 & 11 & 0.0 .9659 \\
\hline & 4 & 1 & 16 & 0.9796 & 1 & 8 & 0.9799 & 1 & 8 & 0.9796 & 1 & 4 & 0.9799 \\
\hline & 6 & 0 & 10 & 0.9576 & 0 & 5 & 0.9573 & 0 & 5 & 0.9576 & 1 & 4 & 0.9967 \\
\hline & 8 & 0 & 10 & 0.9792 & 0 & 5 & 0.9791 & 0 & 5 & 0.9792 & 0 & 3 & 0.9671 \\
\hline \multirow{4}{*}{0.10} & 2 & 8 & 60 & 0.9538 & 8 & 30 & 0.9568 & 8 & 30 & 0.9538 & 8 & 15 & 0.9568 \\
\hline & 4 & 2 & 27 & 0.9844 & 1 & 10 & 009510 & 2 & 14 & 0.981 & 1 & 5 & 0.951 \\
\hline & 6 & 1 & 21 & 0.9884 & 1 & 10 & 0.9908 & 1 & 11 & 0.9858 & 1 & 5 & 0.9908 \\
\hline & 8 & 0 & 13 & 0.9601 & 0 & 7 & 0.9522 & 0 & 7 & 0.9524 & 1 & 5 & 0.9976 \\
\hline \multirow{4}{*}{0.05} & 2 & 10 & 75 & 0.9524 & 11 & 40 & 0.9683 & 11 & 40 & 0.9655 & 11 & 20 & 0.9683 \\
\hline & 4 & 2 & 30 & 0.9726 & 2 & 15 & 0.9731 & 2 & 15 & 0.9726 & 2 & 8 & 0.9625 \\
\hline & 6 & 1 & 24 & 0.9795 & 1 & 12 & 0.9797 & 1 & 12 & 0.9795 & 1 & 6 & 0.9797 \\
\hline & 8 & 1 & 24 & 0.9942 & 1 & 12 & 0.9942 & 1 & 12 & 0.9942 & 1 & 6 & 0.9942 \\
\hline \multirow{4}{*}{0.01} & 2 & 16 & 119 & 0.9563 & 16 & 60 & 0.9557 & 16 & 60 & 0.9514 & 16 & 30 & 0.9557 \\
\hline & 4 & 3 & 45 & 0.9655 & 3 & 23 & 0.9614 & 3 & 23 & 0.9607 & 3 & 12 & 0.9507 \\
\hline & 6 & 2 & 38 & 0.9890 & 2 & 19 & 0.9892 & 2 & 19 & 0.9890 & 2 & 10 & 0.9856 \\
\hline & 8 & 1 & 31 & 0.9820 & 1 & 16 & 0.9796 & 1 & 16 & 0.9795 & 1 & 8 & 0.9796 \\
\hline
\end{tabular}


Table 11. Proposed plan having $w=3$ under Type II GHLD for $25^{\text {th }}$ percentile for $\hat{\theta}=0.6809$.

\begin{tabular}{|c|c|c|c|c|c|c|c|c|c|c|c|c|c|}
\hline \multirow{3}{*}{$\beta$} & \multirow{3}{*}{$t_{q} / t_{q}^{0}$} & \multicolumn{6}{|c|}{$r=5$} & \multicolumn{6}{|c|}{$r=10$} \\
\hline & & \multicolumn{3}{|c|}{$\delta_{q}^{0}=0.5$} & \multicolumn{3}{|c|}{$\delta_{q}^{0}=1.0$} & \multicolumn{3}{|c|}{$\delta_{q}^{0}=0.5$} & \multicolumn{3}{|c|}{$\delta_{q}^{0}=1.0$} \\
\hline & & $c$ & $\mathrm{~g}$ & pa & $c$ & $\mathrm{~g}$ & pa & c & $\mathrm{g}$ & pa & $c$ & $\mathrm{~g}$ & $\mathrm{~Pa}$ \\
\hline \multirow{4}{*}{10.25} & 2 & 5 & 15 & 0.9674 & 6 & 9 & 0.9649 & 6 & 9 & 0.9663 & - & - & - \\
\hline & 4 & 1 & 7 & 0.9748 & 1 & 3 & 0.9867 & 1 & 4 & 0.9571 & 1 & 2 & 0.9557 \\
\hline & 6 & 0 & 4 & 0.9611 & 0 & 2 & 0.9597 & 0 & 2 & 0.9611 & 0 & 1 & 0.9597 \\
\hline & 8 & 0 & 4 & 0.9812 & 0 & 2 & 0.9806 & 0 & 2 & 0.9812 & 0 & 1 & 0.9806 \\
\hline \multirow{4}{*}{0.10} & 2 & 8 & 24 & 0.9655 & 8 & 12 & 0.9638 & 8 & 12 & 0.9655 & - & - & - \\
\hline & 4 & 1 & 8 & 0.9571 & 1 & 4 & 0.9557 & 1 & 4 & 0.9571 & 1 & 2 & 0.9557 \\
\hline & 6 & 1 & 8 & 0.9923 & 1 & 4 & 0.9923 & 1 & 4 & 0.9923 & 1 & 2 & 0.9923 \\
\hline & 8 & 1 & 8 & 0.998 & 1 & 4 & 0.998 & 1 & 4 & 0.9980 & 1 & 2 & 0.998 \\
\hline \multirow{4}{*}{0.05} & 2 & 9 & 28 & 0.9504 & 10 & 15 & 0.9636 & 9 & 14 & 0.9504 & - & - & - \\
\hline & 4 & 2 & 12 & 0.9777 & 2 & 6 & 0.9772 & 2 & 6 & 0.9777 & 2 & 3 & 0.9772 \\
\hline & 6 & 1 & 10 & 0.9796 & 1 & 5 & 0.9793 & 1 & 5 & 0.9796 & 1 & 3 & 0.9567 \\
\hline & 8 & 1 & 10 & 0.9943 & 1 & 5 & 0.9943 & 1 & 5 & 0.9943 & 1 & 3 & 0.9869 \\
\hline \multirow{4}{*}{0.01} & 2 & 14 & 43 & 0.9555 & 13 & 20 & 0.9512 & 15 & 23 & 0.9564 & - & - & - \\
\hline & 4 & 3 & 18 & 0.9728 & 3 & 9 & 0.9721 & 3 & 9 & 0.9728 & 4 & 5 & 0.9925 \\
\hline & 6 & 1 & 12 & 0.9576 & 1 & 6 & 0.9567 & 1 & 6 & 0.9576 & 1 & 3 & 0.9567 \\
\hline & 8 & 1 & 12 & 0.9871 & 1 & 6 & 0.9869 & 1 & 6 & 0.9871 & 1 & 3 & 0.9869 \\
\hline
\end{tabular}

Table 12. Proposed plan having $w=3$ under Type II GHLD for $10^{\text {th }}$ percentile for $\hat{\theta}=0.6809$.

\begin{tabular}{|c|c|c|c|c|c|c|c|c|c|c|c|c|c|}
\hline \multirow{3}{*}{$\beta$} & \multirow{3}{*}{$t_{q} / t_{q}^{0}$} & \multicolumn{6}{|c|}{$r=5$} & \multicolumn{6}{|c|}{$r=10$} \\
\hline & & \multicolumn{3}{|c|}{$\delta_{q}^{0}=0.5$} & \multicolumn{3}{|c|}{$\delta_{q}^{0}=1.0$} & \multicolumn{3}{|c|}{$\delta_{q}^{\mathrm{o}}=0.5$} & \multicolumn{3}{|c|}{$\delta_{q}^{0}=1.0$} \\
\hline & & $c$ & $\mathrm{~g}$ & pa & $c$ & G & pa & $c$ & $\mathrm{~g}$ & pa & $c$ & $\mathrm{~g}$ & $\mathrm{~Pa}$ \\
\hline \multirow{4}{*}{0.25} & 2 & 5 & 38 & 0.9641 & 4 & 16 & 0.9512 & 5 & 19 & 0.9641 & 4 & 8 & 0.9512 \\
\hline & 4 & 1 & 16 & 0.9826 & 1 & 8 & 0.9825 & 1 & 8 & 0.9826 & 1 & 4 & 0.9825 \\
\hline & 6 & 0 & 10 & 0.9615 & 0 & 5 & 0.9609 & 0 & 5 & 0.9615 & 1 & 4 & 0.9972 \\
\hline & 8 & 0 & 10 & 0.9813 & 0 & 5 & 0.9811 & 0 & 5 & 0.9813 & 0 & 3 & 0.9700 \\
\hline \multirow{4}{*}{0.10} & 2 & 8 & 61 & 0.960 & 7 & 27 & 0.955 & 8 & 31 & 0.9536 & 8 & 15 & 0.9645 \\
\hline & 4 & 2 & 27 & 0.9874 & 1 & 10 & 0.9567 & 2 & 14 & 0.9846 & 1 & 5 & 0.9567 \\
\hline & 6 & 1 & 21 & 0.9903 & 1 & 10 & 0.9922 & 1 & 11 & 0.9981 & 1 & 5 & 0.9922 \\
\hline & 8 & 0 & 14 & 0.9568 & 0 & 7 & 0.9564 & 0 & 7 & 0.9568 & 1 & 5 & 0.9980 \\
\hline \multirow{4}{*}{0.05} & 2 & 10 & 77 & 0.9551 & 10 & 38 & 0.9551 & 11 & 41 & 0.9682 & 10 & 19 & 0.9591 \\
\hline & 4 & 2 & 31 & 0.9733 & 2 & 15 & 0.9773 & 2 & 16 & 0.9686 & 2 & 8 & 0.9682 \\
\hline & 6 & 1 & 24 & 0.9827 & 1 & 12 & 0.9826 & 1 & 12 & 0.9827 & 1 & 6 & 0.9826 \\
\hline & 8 & 1 & 24 & 0.9952 & 1 & 12 & 0.9952 & 1 & 12 & 0.9952 & 1 & 6 & 0.9952 \\
\hline \multirow{4}{*}{0.01} & 2 & 15 & 116 & 0.9523 & 14 & 54 & 0.9514 & 15 & 58 & 0.9523 & 14 & 27 & 0.9514 \\
\hline & 4 & 3 & 46 & 0.9686 & 3 & 23 & 0.9682 & 3 & 23 & 0.9686 & 3 & 12 & 0.9590 \\
\hline & 6 & 2 & 39 & 0.9899 & 2 & 19 & 0.9913 & 2 & 20 & 0.9883 & 2 & 10 & 0.9882 \\
\hline & 8 & 1 & 32 & 0.9827 & 1 & 16 & 0.9826 & 1 & 16 & 0.9827 & 1 & 8 & 0.9826 \\
\hline
\end{tabular}


Table 13. Comparison of proposed plan with ordinary group plan with $\theta=1.5, \delta_{q}^{0}=0.5$ and $r=5$ for $25^{\text {th }}$ percentile.

\begin{tabular}{ccrccc}
\hline \multicolumn{2}{c}{$\begin{array}{c}\text { Proposed group } \\
\text { Sampling plan }\end{array}$} & \multicolumn{2}{c}{$\begin{array}{c}\text { Ordinary group } \\
\text { Sampling plan }\end{array}$} \\
\hline$t_{q} / t_{q}^{0} \mathrm{w}=2$ & & $\mathrm{w}=3$ & $\mathrm{~g}$ & $\mathrm{c}$ \\
& $\mathrm{g}, \mathrm{c}$ & $\mathrm{g}, \mathrm{c}$ & 25 & 13 \\
\hline 2 & 17,7 & 15,5 & 8 & 3 \\
4 & 8,2 & 6,1 & 6 & 2 \\
6 & 6,1 & 4,1 & 6 & 2 \\
8 & 3,0 & 4,1 & & \\
\hline
\end{tabular}

\section{Application of the Proposed RGASP with Real Data Set}

In this section, the proposed Type-II GHLD is illustrated with following real lifetime data on clean up gradient ground-water monitoring wells in $(\mu \mathrm{g} / \mathrm{L})$ from vinyl chloride data $[38,39]$.

$0.1,0.1,0.2,0.2,0.4,0.4,0.4,0.5,0.5,0.5,0.6,0.6,0.8,0.9,0.9,1.0,1.1,1.2,1.2$, $1.3,1.8,2.0,2.0,2.3,2.4,2.5,2.7,2.9,3.2,4.0,5.1,5.3,6.8,8.0$.

Assume that the data set follows the Type II Generalized half logistic distribution. The MLEs of the parameters are obtained as $\hat{\theta}=0.6809$. To test the goodness of fit we apply the Kolmogorov-Smirnov test, it is observed that for the data set KolmogorovSmirnov statistic is 0.15704 with p-value is 0.4224 . Thus, the data set is reasonably fitted for Type II GHLD. Figure 1 display Superimposed Empirical, Theoretical Density plots and Q-Q Plot, which shows that Type II GHLD well fitted for this data set.

Assume that lifetime of the products follows Type II generalized half Logistic distribution. An experimenter would like to establish $25^{\text {th }}$ percentile lifetime for the vinyl chloride data for cleanup gradient ground-water monitoring wells through the experiment to be completed for vinyl chloride data by 0.4 at $\alpha=0.05$. The experimenter wants to adopt the proposed plan having $r=5$ for resubmitted lot with $w=2$. Since $\hat{\theta}=0.6809$, the consumer's risk is $25^{\text {th }}$ lifetime percentile, from Table 9 at $t_{q} / t_{q}^{0}=4, \beta=0.25$ and $\delta_{q}$ $=0.5$, the minimum number of groups and acceptance numbers $g=8, c=2$. Does the proposed sampling plan is implemented as follows: select 40 items at random and allocate 5 items to each of 8 groups. Accept the lots if the number of failures is less than or equal two from 8 groups, the product is not accepted then apply the proposed resubmitted acceptance sampling plan for the second time. Accept the lot, if the number of failures is not more than two from the second sample; otherwise reject the same lot. 

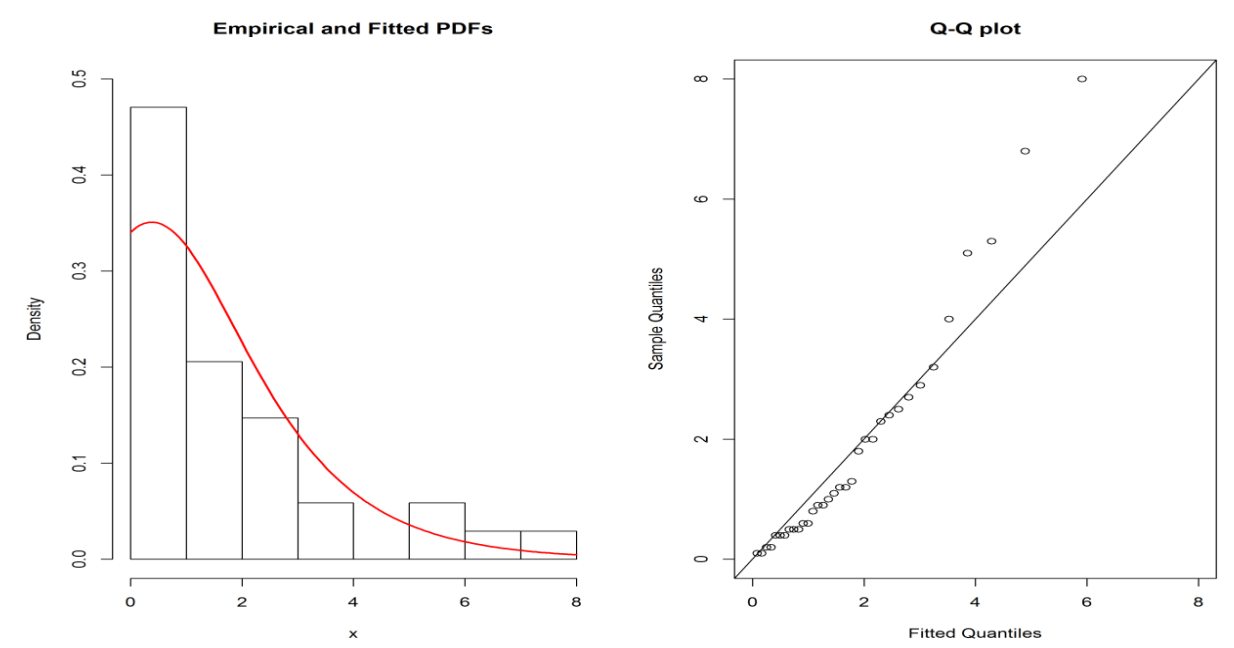

Fig. 1. Superimposed Empirical, Theoretical Density plots and Q-Q Plot of the fitted Type II GHLD for vinyl chloride data.

\section{Comparison study}

The proposed group sampling plan is a generalization of the ordinary group sampling plan under a truncated life test. In this comparison study, comparisons between the proposed group sampling plan and the ordinary group sampling plan are conducted. We present a comparison of the proposed sampling plans based on $w=2,3$ with the $\beta=0.25, \delta_{q}=0.5$ and $r=5$ in Table 13. From Table 13, it can be seen that for a given value of $t_{q} / t_{q}^{0}$, the proposed group sampling plan parameters are always less than the corresponding plan parameters from the ordinary group sampling plan. For example, for a given value $t_{q} / t_{q}^{0}$ $=2$, the proposed group acceptance sampling plan needed $c=7$ and $g=17$ for $w=2$ and $c=5$ and $g=15$ for $w=3$. However, the ordinary group sampling plan requires $c=13$ and $g=25$.

\section{Conclusion}

Usually, an industrial engineer or an experimenter would like to use a sampling plan, which could save time and cost for the life test. In this paper, a group sampling plan to ensure the specified product lifetime percentile for s resubmitted lot has been developed for Type II Generalized half logistic distribution. In this proposed sampling plan, the parameters $\mathrm{c}$ and $\mathrm{g}$ are determined by two-point method. In other words, these parameters are determined such that the lot acceptance probability is large than $1-\alpha$ at the producerspecified quality level but the lot acceptance probability is similar than $\beta$ at the consumer-specified quality level. Considerable tables have been provided for the industrial use according to different parameters and percentile values. It was observed that the number of groups required increases as the consumer's confidence increases, true quality decreases. More importantly, the sample size can be significantly reduced by 
allowing just a small number of resubmissions in the proposed sampling plan. A comparison between the proposed group sampling plan and the ordinary group acceptance sampling plan has also been discussed. It has been noticed that the proposed plan requires a smaller sample size than the ordinary group sampling plan does. Using Bayesian approach and cost model, we can also extend the study to other lifetime distributions.

\section{References}

1. B. Epstein, Annals Math. Stat. 25, 555 (1954). https://www.jstor.org/stable/2236837

2. M. Sobel and J. A. Tischendrof, Philadelphia PA (1959).

3. H. P. Goode and J. H. K. Kao, Philadelphia, PA (1961).

4. S. S. Gupta and P. A. Groll, J. Am. Stat. Assoc. 56, 942 (1961) https://doi.org/10.1080/01621459.1961.10482137

5. S. S. Gupta, Technometrics 4, 2 (1962). https://doi.org/10.1080/00401706.1962.10490002

6. K. W. Fertig and N. R. Mann, Technometrics 22 (1980). https://doi.org/10.2307/1268387

7. R. R. L. Kantam and K. Rosaiah, IAPQR Transactions 23, 2 (1998).

8. R. R. L. Kantam, K. Rosaiah, and G. S. Rao, J. Appl. Stat. 28, 1 (2001). https://doi.org/10.1080/02664760120011644

9. A. Baklizi, Advances Applications Stat. 3, 1(2003) https://doi.org/10.1111/j.0272-4332.2004.00541.x

10. C. J. Wu and T. R. Tsai, Int. J. Reliability, Quality Safety Eng. 12, 507 (2005). https://doi.org/10.1142/S0218539305001987

11. K. Rosaiah and R. R. L. Kantam, Economic Quality Control 20, 2 (2005). https://doi.org/10.1515/EQC.2005.277

12. T. R. Tsai and S. J. Wu, J. App. Stat. 33, 595 (2006). https://doi.org/10.1080/02664760600679700

13. C. H. Jun, S. Balamurali, and S. H. Lee, IEEE Transact. Reliability 55 (2006) https://doi.org/10.1109/TR.2005.863802

14. M. Aslam and C.-H. Jun, J. Appl. Stat. 36, 1021 (2009). https://doi.org/10.1080/02664760802566788

15. M. Aslam, C. -H. Jun, and M. Ahmad, Pak. J. Stat. 25, 3(2009).

16. G. S. Rao, Economic Quality Control 24, 75 (2009).

17. G. S. Rao, Statistica Applicazioni VIII, (2010).

18. N. Balakrishnan, V. Leiva, and J. López, Commun. Stat.-Simul. Comput. 36, 3 (2007). https://doi.org/10.1080/03610910701207819

19. Y. L. Lio, T. R. Tsai, and S. J. Wu, Commun.Stat.-Simul. Comput. 39, 119 (2009). https://doi.org/10.1080/03610910903350508

20. Y. L. Lio, T. R. Tsai, and S. J. Wu, J. Chinese Inst. Indust. Engineers 27, 270 (2010). https://doi.org/10.1080/10170661003791029

21. G. S. Rao and R. R. L. Kantam, Economic Quality Control 25, 153 (2010). https://doi.org/10.1515/eqc.2010.008

22. G. S. Rao, Brazil. J. Probability Stat. 27, 117 (2013). https://doi.org/10.1214/11-BJPS145

23. K. Govindaraju and S. Ganesalingam, Commun. Stat.-Simul. Comput. 26, 1163 (1997). https://doi.org/10.1080/03610919708813433

24. M. Aslam, C. -H. Jun, H. Lee, M. Ahmad, and M. Rasool, Chil. J. Stat. 2, 85 (2011).

25. M. Aslam, M. Azam, and C. -H. Jun, J. Proc. Cont. 24, 1149 (2014). https://doi.org/10.1016/j.jprocont.2014.05.001 
26. S. W. Liu and C. W. Wu, Commun. Stat.-Simul. Comput. 43, 1166 (2014). https://doi.org/10.1080/03610918.2013.810258

27. S. W. Liu, S. W. Lin, and C. W. Wu, Int. J. Prod. Res. 52, 3744 (2014). https://doi.org/10.1080/00207543.2014.886028

28. G. S. Rao and B. S. Rao, J. Data Sci. 12, (2014).

29. C. J. Wu, M. Aslam, C. C. James, and C. H. Jun, Eur. J. Indust. Eng. 9 (2015). https://doi.org/10.1504/EJIE.2015.069340

30. A. R Mughal, Z. Zain, and N. Aziz, Res. J. Appl. Sci. Eng. Technol. 10, 599 (2015). https://doi.org/10.19026/rjaset.10.2468

31. C. H Yen, C. H. Chang, and M. Aslam, J. Stat. Comput. Simul. 85, 1102 (2015). https://doi.org/10.1080/00949655.2013.862791

32. G. S. Rao and C. N. Ramesh, J. Indust. Prod. Eng. 33, 114 (2016). http://dx.doi.org/10.1080/21681015.2015.1111265

33. G. S. Rao and M. Aslam, Commun. Stat.-Simul. Comput. 46, 1814 (2017). https://doi.org/10.1080/03610918.2015.1016236

34. K. Rosaiah, G. S. Rao, K. Kalyani, and D. C. U. S. kumar, Int. J. Quality Reliability Management 34, 1343 (2017). https://doi.org/10.1108/IJQRM-01-2016-0013

35. J. -Y. Chiang, X. Zhao, L.Yang, X. Wang, T. Wang, and H. Zhao, Int. J. Intelligent Technol. Appl. Stat. 11, 57 (2018). http://dx.doi.org/10.6148\%2fIJITAS.201803_11(1).0004

36. G. S. Rao, K. Rosaiah, and M. S. Babu, Int. J. Comput. Sci. Math. 10 (2019). http://dx.doi.org/10.1504/IJCSM.2019.097633

37. R. R. L. Kantam, V. Ramakrishna, and M. S. Ravikumar, Modern Appl. Stat. Methods $\mathbf{1 3}$ (2014). https://doi.org/10.22237/jmasm/1398917760

38. D. K. Bhaumik and R. D. Gibbons, Technometrics 48, 112 (2006). https://doi.org/10.1198/004017005000000355

39. K. Krishnamoorthy, T. Mathew, and S. Mukherjee, Technometrics 50, 69 (2008). https://doi.org/10.1198/004017007000000353 